\title{
Integrality and comprehensiveness of service provision in Primary Health Care in Brazil (2012-2018)
}

\author{
Integralidade e abrangência da oferta de serviços na Atenção Básica no Brasil (2012-2018) \\ Integralidad e integralidad de la prestación de servicios en Atención Primaria en Brasil (2012-2018)
}

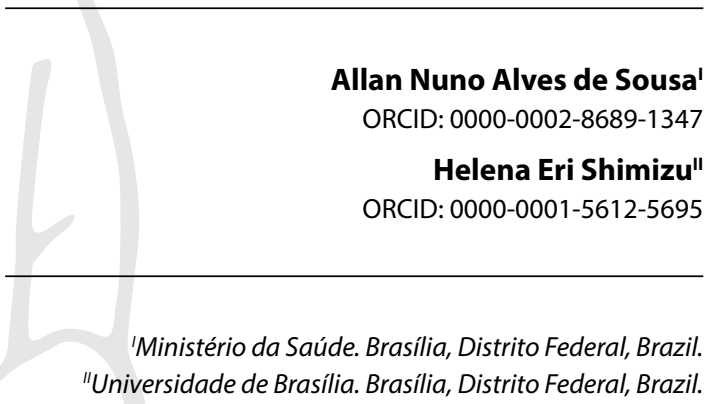

How to cite this article:

Sousa ANA, Shimizu HE. Integrality and comprehensiveness of service provision in Primary Health Care in Brazil

(2012-2018). Rev Bras Enferm. 2021;74(2):e20200500. https://doi.org/10.1590/0034-7167-2020-0500

Corresponding author:

Allan Nuno Alves de Sousa E-mail: alnunos@gmail.com

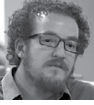

EDITOR IN CHIEF: Antonio José de Almeida Filho ASSOCIATE EDITOR: Ana Fátima Fernandes

Submission: $08-07-2020$

Approval: 10-03-2020

\begin{abstract}
Objectives: to analyze comprehensiveness elements in Primary Care in Brazil, between 2012 and 2018, considering preventive and assistance aspects, pointing out advances and obstacles to its improvement in different contexts. Methods: a retrospective longitudinal study using data from the Brazilian National Program for Improving Access and Quality in Primary Care. 15,378 teams were selected that participated in both $1^{\text {st }}$ and $3^{\text {rd }}$ cycles of the program. Results: improvements were found in the prevalence of teams that ensure preventive and assistance care for priority groups, who develop promotion actions, offer essential procedures, including oral health, carry out home visits, receive support from Extended Family Health and Primary Care Center, offer integrative and complementary practices and develop intersectoral actions. Conclusions: there has been an improvement in comprehensiveness in Primary Health services, but problems remain that still need to be faced for their improvement.

Descriptors: Primary Health Care; Comprehensive Health Care; Health Evaluation; Health Policy; Health Services.
\end{abstract}

\section{RESUMO}

Objetivos: analisar elementos da integralidade na Atenção Básica no Brasil, entre os anos de 2012 e 2018, considerando aspectos preventivos e assistenciais, apontando avanços e obstáculos para a sua melhoria, em diferentes contextos. Métodos: estudo longitudinal retrospectivo utilizando dados do Programa Nacional de Melhoria do Acesso e da Qualidade da Atenção Básica. Foram selecionadas 15.378 equipes que participaram tanto do $1^{\circ}$ quanto do $3^{\circ}$ ciclo do programa. Resultados: constataram-se melhorias na prevalência de equipes que asseguram cuidados preventivos e assistenciais para grupos prioritários, que desenvolvem ações de promoção, ofertam procedimentos essenciais, incluindo saúde bucal, realizam visitas domiciliares, recebem apoio do Núcleo Ampliado de Saúde da Família e Atenção Básica, ofertam práticas integrativas e complementares e desenvolvem ações intersetoriais. Conclusões: houve melhoria da integralidade nos serviços básicos de saúde, mas permanecem problemas que ainda precisam ser enfrentados para o seu aperfeiçoamento. Descritores: Atenção Primária à Saúde; Assistência Integral à Saúde; Avaliação em Saúde; Política de Saúde; Serviços de Saúde.

\section{RESUMEN}

Objetivos: analizar los elementos de la integralidad en la atención primaria en Brasil, entre los años 2012 y 2018, considerando aspectos preventivos y asistenciales, señalando avances y obstáculos para su mejora, en diferentes contextos. Métodos: estudio longitudinal retrospectivo con datos del Programa Nacional de Mejoramiento del Acceso y la Calidad en Atención Primaria. Se seleccionaron 15,378 equipos que participaron tanto en el primer como en el tercer ciclo del programa. Resultados: hubo mejoras en la prevalencia de equipos que aseguran la atención preventiva y asistencial a los grupos prioritarios, que desarrollan acciones de promoción, ofrecen procedimientos esenciales, incluida la salud bucal, realizan visitas domiciliarias, reciben apoyo del Centro de Salud de la Familia Extendida y Atención Primaria, ofrecer prácticas integradoras y complementarias y desarrollar acciones intersectoriales. Conclusiones: hubo una mejora en la integralidad de los servicios básicos de salud, pero persisten problemas que aún deben ser afrontados para su mejora.

Descriptores: Atención Primaria de Salud; Atención Integral de Salud; Evaluación en Salud; Política de Salud; Servicios de Salud. 


\section{INTRODUCTION}

The construction of the Unified Health System (SUS - Sistema Único de Saúde) institutionalized a triad of principles, universality, integrality, and equity, which began to constrain the space to formulate and implement health policies and programs that, from then on, began to constitute themselves ${ }^{(1-2)}$. Comprehensiveness refers to a set of attributes and values that deserve to be defended in the health system, based on the articulation of preventive and assistance services that act to contribute to transform the social framework that affects the population's general health conditions and ensure an assistance offer that is adjusted and compatible with the demands and needs of individuals who seek health services ${ }^{(3)}$.

Comprehensiveness must involve not only individual therapeutic practices, but also collective and collectivizing practices that permeate the health-disease process, in order to ensure desirable conditions of health well-being for the population ${ }^{(1)}$. Primary care (PC), organized based on territorial bases, represents a privileged locus for establishing important elements of integrality, mainly due to its proximity to the spaces in which individuals live, interact and build a good part of relationships with each other and with the environment in which they live ${ }^{(4)}$.

In Brazil, PC was constituted heterogeneously, with diversified ways of structuring and developing actions. But it was with the implementation of Family Health (FH that the country started to have more outlined outlines of a PC organization model guided by a comprehensive concept, which understands it as part of the social and economic development process ${ }^{(5)}$. Since then, as an important element for the effectiveness of comprehensiveness, access to PC has gained greater momentum and has experienced a period of rapid expansion of population coverage under Family Health Strategy (FHS), rising from $6.5 \%$ in 1998 to $64.2 \%$ in $2018^{(6)}$.

As a result of this increase in coverage and PC qualification, several studies have brought evidence on the results produced by FHS, revealing its ability to produce effects on the population's health conditions, with improved access to preventive and assistance services, reducing inequities and increasing SUS efficiency, in addition to contributing to reducing preventable hospitalizations ${ }^{(7-8)}$. However, studies also show that, in spite of the good results, challenges still remain for the effectiveness of comprehensiveness in PC through the provision of care aimed at priority population groups ${ }^{(9-10)}$; health promotion and disease prevention actions ${ }^{(11-12)}$; procedures with a wide scope of practices ${ }^{(13-14)}$; oral health supply ${ }^{(15-16)}$; actions performed at users' homes ${ }^{(17-18)}$; support and articulation of actions between different professional categories ${ }^{(17,19)}$; employing a comprehensive range of assistive technologies ${ }^{(20-21)}$; intersectoral actions ${ }^{(12,22)}$.

\section{OBJECTIVES}

To analyze elements of comprehensiveness in primary care in Brazil, between 2012 and 2018, considering preventive and assistance aspects, pointing out advances and obstacles to its improvement in different contexts.

\section{METHODS}

\section{Ethical aspects}

The study used secondary public domain data made available by the Ministry of Health $(\mathrm{MoH})^{(23)}$, without identifying the respondents, therefore, without the need for an opinion issued by the ethics committee.

\section{Design, place of study, and period}

This is a longitudinal, descriptive, retrospective study, with an organized report based on the use of the STrengthening the Reporting of OBservational studies in Epidemiology (STROBE). Secondary databases from the Brazilian National Program for the Improvement of Access and Quality of Primary Care (PMAQ Programa Nacional de Melhoria do Acesso e Qualidade da Atenção Básica), from the $1^{\text {st }}(2012)$ and $3^{\text {rd }}(2018)$ cycles, available on the $\mathrm{MoH}$ website, were used ${ }^{(23)}$.

Data, in the PMAQ's $1^{\text {st }}$ cycle, were collected between May and December 2012 in 17,482 teams, and 65,391 users who were in a Basic Health Unit (BHU) at the time of collection were interviewed, chosen at random. For the $3^{\text {rd }}$ cycle, data collection was carried out between July 2017 and August 2018 in 38,865 teams, and 140,444 users were interviewed. Data collection was carried out by 41 teaching and research institutions, led by Universidade Federal de Pelotas (UFPel), Universidade Federal de Minas Gerais (UFMG), Fundação Oswaldo Cruz (Fiocruz), Universidade Federal da Bahia (UFBA), Universidade Federal de Sergipe (UFS), Universidade Federal do Piauí (UFPI), Universidade Federal do Rio Grande do Norte (UFRN), Universidade Federal do Mato Grosso do Sul (UFMS), Universidade Federal do Pará (UFPA). Researchers from the institutions that led the data collection process were also responsible for developing the instruments and protocols and methodologies used in the field work, in articulation with $\mathrm{MoH}$ technicians.

\section{Sample, inclusion and exclusion criteria}

This is a longitudinal, retrospective study of a sample of 15,378 teams that participated in the $1^{\text {st }}$ and $3^{\text {rd }}$ cycles of the PMAQ, in order to allow an analysis that identifies changes in the behavior of the dimensions related to service offer integrality and comprehensiveness for the same cases over time. Only the teams participating in the two cycles studied were considered to ensure analysis of the evolution of the situation of the same teams regarding the observed aspects. Thus, 2,104 teams participating in the $1^{\text {st }} \mathrm{cycle}$ and 23,487 participants in the $3^{\text {rd }}$ cycle were excluded. We sought to analyze whether for each selected variable there was an improvement, worsening or maintenance of the observed setting. The $\mathrm{BHU}$ results were replicated for all teams that worked in the same $\mathrm{BHU}$, and 59,354 users were selected who responded to the instruments referring to the teams that comprise the sample in the $1^{\text {st }}$ cycle and 56,369 users in the $3^{\text {rd }}$ cycle.

\section{Study protocol}

Variables associated with diverse elements of comprehensiveness in PC were selected considering preventive and care aspects 
and that kept correspondence and content compatibility, since the instruments used in the analyzed cycles were not identical. Many questions remained the same, others suffered adaptations in their statement or in the available alternatives, and in some situations new questions were added and others were removed from the instruments. Thus, to ensure comparability of variables between the periods studied, only questions were selected in which consistency was found in their object and meaning. Altogether 9 variables were chosen that refer to information from the teams, such as provision of programmed actions for priority population groups, development of promotion and prevention actions, performance of procedures and minor surgeries, offer of oral health, carrying out actions at families' homes and developing intersectoral actions; 6 variables deal with users' position regarding the topics covered, such as adequate provision of care to priority groups, provision and guidance on the use of dental prosthesis and home visits.

In addition to the comparative analysis between 2012 and 2018 , data were stratified by region and population size of the municipalities. For population size, the municipalities were stratified based on the population estimate of the Brazilian Institute of Geography and Statistics (IBGE - Instituto Brasileiro de Geografia e Estatística) for 2012 (up to 10,000 inhabitants, from 10,001 to $20,000,20,001$ to $50,000,50,001$ to 100,000 and above 100,000 inhabitants).

\section{Analysis of results, and statistics}

Data were organized and treated in a Microsoft Office Excel Professional Plus $2013^{\circ}$ spreadsheet. For data analysis referring to the comprehensiveness and scope of service offer in crossing with region and population size, the chi-square test was applied for expected proportions equal in samples of nominal variables, and all $p$ value values below 0.05 were considered statistically significant.

\section{RESULTS}

Between 2012 and 2018, the proportion of teams offering scheduled actions for priority population groups increased from $70.7 \%$ to $93.3 \%$, with greater emphasis on the south and center-west, with increases of 27.8 and 27.0 p.s., respectively, and in municipalities with up to 20 thousand inhabitants (Table 1). When it comes to promotion and prevention actions, the increase was even more expressive, rising from $68.1 \%$ in 2012 and reaching $96.2 \%$ in 2018, with an increase of more than 30 p.s. in the southeast, south and center-west and in municipalities with up to 50 thousand inhabitants (Table 1).

The prevalence of teams that collect material for laboratory tests increased by 12.1 p.s., reaching almost $2 / 3$ of the total teams that make up the sample (62.6\%), with greater increases in the south (20.6 p.s.) and municipalities between 10 and 20 thousand inhabitants (20.7 p.s.). At the same time, the increase in the number of teams that perform an essential list of procedures and minor surgeries was 26.2 p.s.. The northeast and central-west showed the best advances. Concerning the size of municipalities, it was in the locations with up to 50 thousand inhabitants that the most marked improvements occurred (Table 1). For oral health, the national increase in the prevalence of teams that carry out a basic list of procedures was only 1.4 p.s., with an increase only for the northeast and greater improvements in municipalities with between 20 and 100 thousand inhabitants (Table 1). With regard to home visits, the prevalence of teams that organize to visit families and users, according to risk and vulnerability, was already high in 2012 (92.9\%), increasing to 96.0\% in 2018, with greater growth in the center-west and a small decrease for municipalities above 100 thousand inhabitants (Table 1).

Between 2012 and 2018, the proportion of teams that had the support of the Family Health and Primary Care Extended Centers (NASF - Núcleo Ampliado de Saúde da Família e Atenção Básica) increased from $56.5 \%$ to $75.2 \%$, with an increase of more than 30

Table 1 - Integrality and comprehensiveness of service provision in Primary Care according to health professionals, by large region and population size, Brazil, 2012 and 2018

\begin{tabular}{|c|c|c|c|c|c|c|}
\hline \multirow{2}{*}{ Variables } & \multicolumn{2}{|c|}{$\begin{array}{l}\text { Offer programmed actions for } \\
\text { priority population groups }\end{array}$} & \multicolumn{2}{|c|}{$\begin{array}{l}\text { Develop promotion and } \\
\text { prevention action } s^{*(m)}\end{array}$} & \multicolumn{2}{|c|}{$\begin{array}{l}\text { Perform material collection } \\
\text { for laboratory exams*(n) }\end{array}$} \\
\hline & $\begin{array}{c}2012 \\
\%(95 \% \mathrm{Cl})\end{array}$ & $\begin{array}{c}2018 \\
\%(95 \% \mathrm{Cl})\end{array}$ & $\begin{array}{c}2012 \\
\%(95 \% \mathrm{Cl})\end{array}$ & $\begin{array}{c}2018 \\
\%(95 \% \mathrm{Cl})\end{array}$ & $\begin{array}{c}2012 \\
\%(95 \% \mathrm{Cl})\end{array}$ & $\begin{array}{c}2018 \\
\%(95 \% \mathrm{Cl})\end{array}$ \\
\hline \multicolumn{7}{|l|}{ Region } \\
\hline North ${ }^{(a)}$ & $71.8(68.9-74.7)$ & $86.7(84.5-88.9)$ & $78.2(75.5-80.9)$ & $94.2(92.7-95.7)$ & $50.6(47.3-53.9)$ & $44.8(41.6-48.0)$ \\
\hline Northeast $^{(b)}$ & $79.1(78.0-80.2)$ & $95.5(94.9-96.1)$ & $77.8(76.7-78.9)$ & $96.8(96.3-97.3)$ & $35.4(34.1-36.7)$ & $51.2(49.8-52.6)$ \\
\hline Southeast ${ }^{(c)}$ & $65.9(64.7-67.1)$ & $91.8(91.1-92.5)$ & $60.7(59.4-62.0)$ & $95.2(94.6-95.8)$ & $65.0(63.8-66.2)$ & $72.5(71.3-73.7)$ \\
\hline South & $66.1(64.3-67.9)$ & $93.9(93.0-94.8)$ & $63.1(61.2-65.0)$ & $97(96.3-97.7)$ & $44.8(42.9-46.7)$ & $65.4(63.6-67.2)$ \\
\hline Center-West ${ }^{(\mathrm{e})}$ & $68.5(65.7-71.3)$ & $95.5(94.2-96.8)$ & $65.9(63.0-68.8)$ & $98.4(97.6$ - 99.2) & $56.5(53.5-59.5)$ & $71.8(69.1-74.5)$ \\
\hline \multicolumn{7}{|l|}{ Population Size } \\
\hline Up to $10,000^{(f)}$ & $64.9(62.9-66.9)$ & $93.7(92.7-94.7)$ & $66.1(64.1-68.1)$ & $98.6(98.1-99.1)$ & $46.7(44.6-48.8)$ & $63.2(61.2-65.2)$ \\
\hline 10,001 to $20,000^{(\mathrm{g})}$ & $65.3(63.4-67.2)$ & $93.4(92.4-94.4)$ & $65.5(63.6-67.4)$ & $98.6(98.1-99.1)$ & $31.7(29.9-33.5)$ & $52.4(50.4-54.4)$ \\
\hline 20,001 to $50,000^{(\mathrm{h})}$ & $70.9(69.4-72.4)$ & $95.4(94.7-96.1)$ & $67.8(66.2-69.4)$ & $98.4(98.0-98.8)$ & $39.0(37.4-40.6)$ & $56.9(55.2-58.6)$ \\
\hline 50,001 to $100,000^{(i)}$ & $73.0(70.9-75.1)$ & $96.4(95.5-97.3)$ & $68.6(66.4-70.8)$ & $98.2(97.6-98.8)$ & $44.2(41.8-46.6)$ & $59.8(57.4-62.2)$ \\
\hline 100,000 or more ${ }^{(j)}$ & $74.9(73.8-76.0)$ & $91.4(90.7-92.1)$ & $70.1(68.9-71.3)$ & $92.7(92.0-93.4)$ & $69.7(68.5-70.9)$ & $71.5(70.3-72.7)$ \\
\hline $\operatorname{Brazil}^{(k)}$ & $70.7(70.0-71.4)$ & $93.3(92.9-93.7)$ & $68.1(67.4-68.8)$ & $96.2(95.9-96.5)$ & $50.5(49.7-51.3)$ & $62.6(61.8-63.4)$ \\
\hline
\end{tabular}




\begin{tabular}{|c|c|c|c|c|c|c|}
\hline \multirow{2}{*}{ Variables } & \multicolumn{2}{|c|}{$\begin{array}{l}\text { Perform procedures and } \\
\text { minor surgeries }\end{array}$} & \multicolumn{2}{|c|}{$\begin{array}{l}\text { Perform basic oral } \\
\text { health procedures*(p) }\end{array}$} & \multicolumn{2}{|c|}{$\begin{array}{l}\text { Perform home visits according } \\
\text { to risk and vulnerability* }\end{array}$} \\
\hline & $\begin{array}{c}2012 \\
\%(95 \% \mathrm{Cl})\end{array}$ & $\begin{array}{c}2018 \\
\%(95 \% \mathrm{Cl})\end{array}$ & $\begin{array}{c}2012 \\
\%(95 \% \mathrm{Cl})\end{array}$ & $\begin{array}{c}2018 \\
\%(95 \% \mathrm{Cl})\end{array}$ & $\begin{array}{c}2012 \\
\%(95 \% \mathrm{Cl})\end{array}$ & $\begin{array}{c}2018 \\
\%(95 \% \mathrm{Cl})\end{array}$ \\
\hline \multicolumn{7}{|l|}{ Region } \\
\hline North $^{(a)}$ & $17.2(14.7-19.7)$ & $41.1(37.9-44.3)$ & $36.2(33.1-39.3)$ & $30.6(27.6-33.6)$ & $88.3(86.2$ - 90.4) & $93.0(91.3-94.7)$ \\
\hline Northeast $^{(b)}$ & $8.0(7.3-8.7)$ & $45.1(43.7-46.5)$ & $54.2(52.8-55.6)$ & $64.6(63.3-65.9)$ & $93.9(93.2-94.6)$ & $96.6(96.1-97.1)$ \\
\hline Southeast ${ }^{(c)}$ & $8.7(8.0-9.4)$ & $26.8(25.7-27.9)$ & $46.5(45.2-47.8)$ & $43.5(42.2-44.8)$ & $93.1(92.4-93.8)$ & $95.1(94.5-95.7)$ \\
\hline South ${ }^{(d)}$ & $27.9(26.2-29.6)$ & $51.8(49.9-53.7)$ & $48.7(46.8-50.6)$ & $46.9(45.0-48.8)$ & $93.9(93.0-94.8)$ & $96.8(96.1-97.5)$ \\
\hline Center-West $^{(\mathrm{e})}$ & $18.0(15.7-20.3)$ & $44.2(41.2-47.2)$ & $57.5(54.5-60.5)$ & $52.4(49.4-55.4)$ & $87.7(85.7$ - 89.7) & $98.4(97.6$ - 99.2) \\
\hline \multicolumn{7}{|l|}{ Population Size } \\
\hline Up to $10,000^{(f)}$ & $32.1(30.2-34.0)$ & $64.9(62.9-66.9)$ & $56.1(54.0-58.2)$ & $59.5(57.5-61.5)$ & $90.6(89.4$ - 91.8) & $98.3(97.8-98.8)$ \\
\hline 10,001 to $20,000^{(\mathrm{g})}$ & $13.0(11.7-14.3)$ & $45.0(43.0-47.0)$ & $52.3(50.3-54.3)$ & $56.6(54.6-58.6)$ & $91.6(90.5-92.7)$ & $98.4(97.9$ - 98.9) \\
\hline 20,001 to $50,000^{(h)}$ & $9.1(8.1-10.1)$ & $42.2(40.5-43.9)$ & $52.3(50.6-54.0)$ & $60.5(58.8-62.2)$ & $93.2(92.3-94.1)$ & $98.6(98.2$ - 99.0) \\
\hline 50,001 to $100,000^{(i)}$ & $8.4(7.1$ - 9.7) & $35.0(32.7-37.3)$ & $51.7(49.3-54.1)$ & $56.8(54.4-59.2)$ & $90.6(89.2-92.0)$ & $97.9(97.2-98.6)$ \\
\hline 100,000 or more ${ }^{(j)}$ & $8.6(7.9-9.3)$ & $25.5(24.3-26.7)$ & $43.1(41.8-44.4)$ & $37.1(35.8-38.4)$ & $94.9(94.3-95.5)$ & $92.3(91.6-93.0)$ \\
\hline Brazil $^{(\mathrm{k})}$ & $12.9(12.4-13.4)$ & $39.1(38.3-39.9)$ & $49.5(48.7-50.3)$ & $50.9(50.1-51.7)$ & $92.9(92.5-93.3)$ & $96.0(95.7-96.3)$ \\
\hline
\end{tabular}

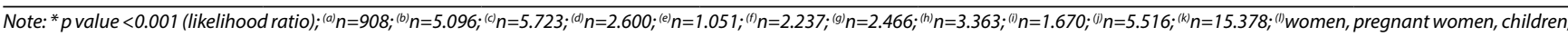
users with hypertension and users with diabetes mellitus; ${ }^{(m)}$ For the $1^{\text {st }}$ cycle, the five variables that showed the best results were selected: (1) prevention and treatment of diabetes mellitus (89.4\%), (2) prevention and treatment of systemic arterial hypertension (89.3\%), (3) pregnant women and postpartum women (87.3\%), (4) women (cervical and breast cancer) (82.1\%) and family planning (78.5\%); (n)Including collection of cytopathological examination; ${ }^{(0)}$ Abscess drainage, wound suture, stitch removal, ear washing, nail extraction, nebulization/inhalation, dressings, intramuscular injectable medications, intravenous injectable medications and application of benzathine penicillin $G$; (p) Sealant application, topical fluoride application, amalgam restoration, composite resin restoration and extraction.

p.s. in the north and 45 p.s. in municipalities with up to 10 thousand inhabitants (Table 2). At the same time, there was a significant increase in the prevalence of teams that started offering Integrative and Complementary Practices (ICP), from $13.3 \%$ to $34.3 \%$, with emphasis on the southeast $(18.7 \%-41.5 \%)$ and for municipalities above 100 thousand inhabitants (23.8\% - 48.6\%) (Table 2). Finally, when it comes to carrying out intersectoral actions, there was an increase of 9.9 p.s. in the prevalence of teams that carried out activities in schools in their area of coverage, with increases of more than 10 p.s. in the northeast, center-west and southeast and in municipalities over 50 thousand inhabitants (Table 2).

With regard to care for priority population groups, between 2012 and 2018, nationally, there was practically no variation in the percentage of puerperal users who reported having received adequate care during the post-partum consultation, although there was an increase of almost 5 p.s. in the northeast. An increase was found for users who answered affirmatively for access to care for children under 2 years old ( 4.6 p.s.) for a set of elements considered essential. Finally, when it comes to recommended care for people with diabetes, the increase was 4.2 p.s., with the highest increase to the northeast. For the three variables analyzed, no statistically significant differences were found when considering the municipalities' population size (Table 3).

When comparing data on preventive health actions for women, it can be noted that there was a positive variation both for the national staff (44.0\% - 51.0\%) and for all observed contexts (Table 3 ). Among users in need of dental prosthesis, for both years analyzed, the percentage of those who claimed to have prosthesis available at $\mathrm{BHU}$ and received guidance on care they should take in its use was very low (5.2\% and 7,9\%), with the best result for the smallest localities that presented a percentage of $18.4 \%$ for 2018 (Table 3). Most users reported receiving a visit from Community Health Workers (CHW) at their home, although the percentages decreased between 2012 and 2018, with the largest negative variations occurring in the southeast and center-west and in municipalities with more than 100 thousand inhabitants (Table 3).

Table 2 - Integrality and comprehensiveness of service provision in Primary Care according to health professionals, by large region and population size, Brazil, 2012 and 2018

\begin{tabular}{|c|c|c|c|c|c|c|}
\hline \multirow{2}{*}{ Variables } & \multicolumn{2}{|c|}{$\begin{array}{l}\text { Receive support from the Family Health and } \\
\text { Primary Care Extended Centers (NASF)* }\end{array}$} & \multicolumn{2}{|c|}{$\begin{array}{l}\text { Offer integrative and } \\
\text { complementary practices* }\end{array}$} & \multicolumn{2}{|c|}{$\begin{array}{l}\text { Perform activities } \\
\text { in schools* }\end{array}$} \\
\hline & $\begin{array}{c}2012 \\
\%(95 \% C I)\end{array}$ & $\begin{array}{c}2018 \\
\%(95 \% \mathrm{Cl})\end{array}$ & $\begin{array}{c}2012 \\
\%(95 \% \mathrm{CI})\end{array}$ & $\begin{array}{c}2018 \\
\%(95 \% \mathrm{CI})\end{array}$ & $\begin{array}{c}2012 \\
\%(95 \% \mathrm{Cl})\end{array}$ & $\begin{array}{c}2018 \\
\%(95 \% \mathrm{Cl})\end{array}$ \\
\hline \multicolumn{7}{|l|}{ Region } \\
\hline North ${ }^{(a)}$ & $42.0(38.8-45.2)$ & $74.0(71.1-76.9)$ & $8.3(6.5-10.1)$ & $17.6(15.1-20.1)$ & $80.7(78.1-83.3)$ & $87.1(84.9-89.3)$ \\
\hline Northeast $^{(b)}$ & $63.4(62.1-64.7)$ & $84.4(83.4-85.4)$ & $7.5(6.8-8.2)$ & $32.5(31.2-33.8)$ & $79.7(78.6$ - 80.8) & $91.4(90.6-92.2)$ \\
\hline Southeast ${ }^{(\mathrm{c})}$ & $59.4(58.1-60.7)$ & $71.6(70.4-72.8)$ & $18.7(17.7-19.7)$ & $41.5(40.2-42.8)$ & $69.8(68.6-71.0)$ & $80.2(79.2-81.2)$ \\
\hline South $^{(d)}$ & $46.6(44.7-48.5)$ & $67.2(65.4-69.0)$ & $13.3(12.0-14.6)$ & $33.3(31.5-35.1)$ & 77.1 (75.5 - 78.7) & $82.7(81.2-84.2)$ \\
\hline Center-West ${ }^{(\mathrm{e})}$ & $45.1(42.1-48.1)$ & $71.1(68.4-73.8)$ & $15.6(13.4-17.8)$ & $21.1(18.6-23.6)$ & $79.0(76.5-81.5)$ & $90.0(88.2-91.8)$ \\
\hline
\end{tabular}




\begin{tabular}{|c|c|c|c|c|c|c|}
\hline \multirow{2}{*}{ Variables } & \multicolumn{2}{|c|}{$\begin{array}{l}\text { Receive support from the Family Health and } \\
\text { Primary Care Extended Centers (NASF)* }\end{array}$} & \multicolumn{2}{|c|}{$\begin{array}{l}\text { Offer integrative and } \\
\text { complementary practices* }\end{array}$} & \multicolumn{2}{|c|}{$\begin{array}{l}\text { Perform activities } \\
\text { in schools* }\end{array}$} \\
\hline & $\begin{array}{c}2012 \\
\%(95 \% \mathrm{CI})\end{array}$ & $\begin{array}{c}2018 \\
\%(95 \% \mathrm{CI})\end{array}$ & $\begin{array}{c}2012 \\
\%(95 \% \mathrm{CI})\end{array}$ & $\begin{array}{c}2018 \\
\%(95 \% \mathrm{CI})\end{array}$ & $\begin{array}{c}2012 \\
\%(95 \% \mathrm{CI})\end{array}$ & $\begin{array}{c}2018 \\
\%(95 \% C I)\end{array}$ \\
\hline \multicolumn{7}{|l|}{ Population Size } \\
\hline Up to $10,000^{(f)}$ & $27.1(25.3-28.9)$ & $74.2(72.4-76.0)$ & $7.1(6.0-8.2)$ & $27.0(25.2-28.8)$ & $84.3(82.8-85.8)$ & $90.0(88.8-91.2)$ \\
\hline 10,001 to $20,000^{(\mathrm{g})}$ & $39.4(37.5-41.3)$ & $75.4(73.7-77.1)$ & $6.6(5.6-7.6)$ & $23.2(21.5-24.9)$ & $80.5(78.9-82.1)$ & $89.7(88.5-90.9)$ \\
\hline 20,001 to $50,000^{(\mathrm{h})}$ & $60.2(58.5-61.9)$ & $82.0(80.7-83.3)$ & $7.7(6.8-8.6)$ & $26.2(24.7-27.7)$ & $79.9(78.5-81.3)$ & $89(87.9-90.1)$ \\
\hline 50,001 to $100,000^{(i)}$ & $68.9(66.7-71.1)$ & $81.6(79.7-83.5)$ & $8.8(7.4-10.2)$ & $30.7(28.5-32.9)$ & $73.9(71.8-76.0)$ & $87.6(86.0-89.2)$ \\
\hline 100,000 or more ${ }^{(j)}$ & 70.6 (69.4 - 71.8) & $70.1(68.9-71.3)$ & $23.8(22.7-24.9)$ & $48.6(47.3-49.9)$ & $67.4(66.2-68.6)$ & $79.0(77.9-80.1)$ \\
\hline Brazil $^{(k)}$ & $56.5(55.7-57.3)$ & $75.2(74.5-75.9)$ & $13.3(12.8-13.8)$ & $34.3(33.5-35.1)$ & $75.5(74.8-76.2)$ & $85.4(84.8-86.0)$ \\
\hline
\end{tabular}

Note: * $p$ value $<0.001$ (likelihood ratio); ${ }^{(a)} n=908 ;{ }^{(b)} n=5.096 ;{ }^{(c)} n=5.723 ;{ }^{(d)} n=2.600 ;\left({ }^{(e)} n=1.051 ;{ }^{(f)} n=2.237 ;{ }^{(g)} n=2.466 ;{ }^{(h)} n=3.363 ;{ }^{(i)} n=1.670 ;\left({ }^{(j)} n=5.516 ;{ }^{(k)} n=15.378\right.\right.$.

Table 3 - Integrality and comprehensiveness of service offer in Primary Care according to users, by large region and population size, Brazil, 2012 and 2018

\begin{tabular}{|c|c|c|c|c|c|c|}
\hline \multirow{2}{*}{ Variables } & \multicolumn{2}{|c|}{$\begin{array}{l}\text { Review of childbirth and } \\
\text { care for women*(1):(m) }\end{array}$} & \multicolumn{2}{|c|}{$\begin{array}{l}\text { Monitoring of children } \\
\text { under } 2 \text { years }{ }^{*(n):(0)}\end{array}$} & \multicolumn{2}{|c|}{$\begin{array}{l}\text { Monitoring people } \\
\text { with diabetes } s^{(p):(q)}\end{array}$} \\
\hline & $\begin{array}{c}2012 \\
\%(95 \% \mathrm{Cl})\end{array}$ & $\begin{array}{c}2018 \\
\%(95 \% \mathrm{Cl})\end{array}$ & $\begin{array}{c}2012 \\
\%(95 \% \mathrm{Cl})\end{array}$ & $\begin{array}{c}2018 \\
\%(95 \% \mathrm{Cl})\end{array}$ & $\begin{array}{c}2012^{* *} \\
\%(95 \% \mathrm{Cl})\end{array}$ & $\begin{array}{c}2018 * \\
\%(95 \% \mathrm{Cl})\end{array}$ \\
\hline \multicolumn{7}{|l|}{ Region } \\
\hline North ${ }^{(a)}$ & $13.3(8.1-18.5)$ & $14.3(10.1-18.5)$ & $17.5(13.8-21.2)$ & $23.2(19.3-27.1)$ & $17.6(13.2-22.0)$ & $20.7(16.1-25.3)$ \\
\hline Northeast $^{(b)}$ & $23.4(20.8-26.0)$ & $28.3(25.8-30.8)$ & $25.1(23.3-26.9)$ & $33.9(31.8-36.0)$ & $21.0(19.0-23.0)$ & $30.8(28.7-32.9)$ \\
\hline Southeast ${ }^{(c)}$ & $23.7(22.0-25.4)$ & $20.6(18.7-22.5)$ & $33.2(31.7-34.7)$ & $33.1(31.3-34.9)$ & $29.9(28.4-31.4)$ & $32.3(30.7-33.9)$ \\
\hline South ${ }^{(\mathrm{d})}$ & $21.8(18.3-25.3)$ & $24.1(20.6-27.6)$ & $28.3(25.1-31.5)$ & $36.9(33.6-40.2)$ & $22.4(19.9-24.9)$ & $27.6(25.2-30.0)$ \\
\hline Center-West ${ }^{(\mathrm{e})}$ & $15.5(12.0-19.0)$ & $12.2(8.4-16.0)$ & $17.8(15.1-20.5)$ & $27.4(23.3-31.5)$ & $24.5(21.1-27.9)$ & $24(20.3-27.7)$ \\
\hline \multicolumn{7}{|l|}{ Population Size } \\
\hline Up to $10,000^{(f)}$ & $24.3(21.0-27.6)$ & $20.4(17.0-23.8)$ & $27.4(24.8-30.0)$ & $36.2(33.0-39.4)$ & $23.9(21.3-26.5)$ & $28.8(25.8-31.8)$ \\
\hline 10,001 to $20,000^{(g)}$ & $23.0(20.0-26.0)$ & $23.5(20.1-26.9)$ & $28.2(25.8-30.6)$ & $33.6(30.6-36.6)$ & $24.6(22.0-27.2)$ & $29.3(26.5-32.1)$ \\
\hline 20,001 to $50,000^{(\mathrm{h})}$ & $21.2(18.7-23.7)$ & $24.5(21.7-27.3)$ & $26.9(24.8-29.0)$ & $32.3(29.9-34.7)$ & $23.0(20.9-25.1)$ & $29.8(27.5-32.1)$ \\
\hline 50,001 to $100,000^{(i)}$ & $21.1(17.6-24.6)$ & $21.7(18.0-25.4)$ & $29.7(26.7-32.7)$ & $33.1(29.7-36.5)$ & $21.6(18.7-24.5)$ & $29.2(26.1-32.3)$ \\
\hline 100,000 or more ${ }^{(j)}$ & $22.3(20.3-24.3)$ & $21.8(19.7-23.9)$ & $28.6(26.9-30.3)$ & $30.9(29.0-32.8)$ & $29.6(28.0-31.2)$ & $31(29.4-32.6)$ \\
\hline Brazil $^{(k)}$ & $22.3(21.1-23.5)$ & $22.4(21.1-23.7)$ & $28.1(27.1-29.1)$ & $32.7(31.5-33.9)$ & $25.9(24.9-26.9)$ & $30.1(29.1-31.1)$ \\
\hline \multirow{2}{*}{ Variables } & \multicolumn{2}{|c|}{$\begin{array}{l}\text { Preventive actions for } \\
\text { Women's Health**(r);(s) }\end{array}$} & \multicolumn{2}{|c|}{$\begin{array}{l}\text { Provision and guidance on the } \\
\text { use of dental prosthesis**(t) }\end{array}$} & \multicolumn{2}{|c|}{$\begin{array}{l}\text { Receive a visit from a Community } \\
\text { Health Worker at home*** }\end{array}$} \\
\hline & $\begin{array}{c}2012 \\
\%(95 \% \mathrm{Cl})\end{array}$ & $\begin{array}{c}2018 \\
\%(95 \% \mathrm{Cl})\end{array}$ & $\begin{array}{c}2012 \\
\%(95 \% \mathrm{Cl})\end{array}$ & $\begin{array}{c}2018 \\
\%(95 \% \mathrm{Cl})\end{array}$ & $\begin{array}{c}2012 \\
\%(95 \% \mathrm{Cl})\end{array}$ & $\begin{array}{c}2018 \\
\%(95 \% \mathrm{Cl})\end{array}$ \\
\hline \multicolumn{7}{|l|}{ Region } \\
\hline North ${ }^{(a)}$ & $44.7(42.8-46.6)$ & $48.5(46.5-50.5)$ & $1.9(0.3-3.5)$ & $2.3(0.6-4.0)$ & $82.2(80.9-83.5)$ & $78.6(77.1-80.1)$ \\
\hline Northeast $^{(b)}$ & $48.3(47.5-49.1)$ & $57.0(56.2-57.8)$ & $2.2(1.6-2.8)$ & $4.1(3.4-4.8)$ & $84.8(84.3-85.3)$ & $84.6(84.1-85.1)$ \\
\hline Southeast ${ }^{(c)}$ & $40.3(39.6-41.0)$ & $46.8(46.0-47.6)$ & $8.9(7.4-10.4)$ & $11.1(9.7-12.5)$ & $87.7(87.3-88.1)$ & $82.5(82.0-83.0)$ \\
\hline South $^{(d)}$ & $47.5(46.3-48.7)$ & $51.4(50.2-52.6)$ & $7.7(6.2-9.2)$ & $12.1(10.2-14.0)$ & $83.5(82.8-84.2)$ & 79.0 (78.2 -79.8) \\
\hline Center-West ${ }^{(\mathrm{e})}$ & $34.4(32.7-36.1)$ & $44.9(43.1-46.7)$ & $8.2(5.0-11.4)$ & $11.7(8.1-15.3)$ & $81.0(79.8-82.2)$ & $75.8(74.5-77.1)$ \\
\hline \multicolumn{7}{|l|}{ Population Size } \\
\hline Up to $10,000^{(f)}$ & $44.8(43.6-46.0)$ & $53.5(52.3-54.7)$ & $11.5(9.7-13.3)$ & $18.4(16.2-20.6)$ & $89.3(88.7-89.9)$ & $88.5(87.8-89.2)$ \\
\hline 10,001 to $20,000^{(g)}$ & $45.4(44.3-46.5)$ & $54.1(53.0-55.2)$ & $6.9(5.4-8.4)$ & $8.7(7.0-10.4)$ & $88.8(88.2-89.4)$ & $87.7(87.0-88.4)$ \\
\hline 20,001 to $50,000^{(h)}$ & $44.0(43.0-45.0)$ & $53.1(52.1-54.1)$ & $3.0(2.1-3.9)$ & $4.6(3.5-5.7)$ & $86.2(85.6-86.8)$ & $84.1(83.5-84.7)$ \\
\hline 50,001 to $100,000^{(i)}$ & $45.4(44.0-46.8)$ & $52.8(51.4-54.2)$ & $2.5(1.3-3.7)$ & $3.6(2.2-5.0)$ & $84.0(83.1-84.9)$ & $78.9(77.9-79.9)$ \\
\hline 100,000 or more ${ }^{(j)}$ & $42.6(41.8-43.4)$ & $46.3(45.5-47.1)$ & $2.8(2.0-3.6)$ & $5.4(4.4-6.4)$ & $81.8(81.3-82.3)$ & $75.7(75.1-76.3)$ \\
\hline $\operatorname{Brazi}^{(k)}$ & $44.0(43.5-44.5)$ & $51.0(50.5-51.5)$ & $5.2(4.6-5.8)$ & $7.9(7.2-8.6)$ & $85.3(85.0-85.6)$ & $81.9(81.6-82.2)$ \\
\hline
\end{tabular}

Note: ${ }^{*}$ p value $<0.001$ for region and p value $>0.05$ for population size (likelihood ratio); ${ }^{*} p$ value $<0.001$ (likelihood ratio); ${ }^{(a)} 2012=3.231 / 2018=3.049 ;{ }^{(b)} 2012=19.653 / 2018=18.427$; ${ }^{(c)} 2012=22.870 / 2018=21.193 ;$

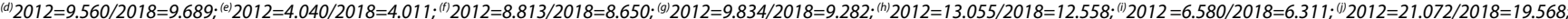
(k) $2012=59.354 / 2018=56.369$; (')Only users who had a postpartum consultation ( $n=4,550$ (2012) and $n=4,058$ (2018)) were considered; ( $m$ ) Issues considered: (1) how the puerperal woman was feeling, if she was experiencing any discouragement, sadness, depression, (2) contraceptive provision, (3) gynecological examination and (4) breast examination; ${ }^{(n)}$ Only users with children up to 2 years old ( $n=7,964$ (2012) and $n=6,183$ (2018)) were considered; ${ }^{(0)}$ Issues considered: (1) actions during the consultation in the first week of life (heavy child, measured, placed for breastfeeding, navel examined and asked if children had a birth certificate), (2) carrying out the heel prick test, (3) up-to-date vaccination, (4) monitoring the child's development and (5) guidance on feeding the child up to two years; ${ }^{(p)}$ Only users with diabetes were considered ( $n=7,359$ (2012) and $n=7,424$ (2018)); ${ }^{(q)}$ Issues considered: in the last 6 months (1), took a blood glucose test, (2) a professional examined the feet, (3) a professional advised about taking care of the feet; ${ }^{(r)}$ Only female users were considered ( $n=46,080(2012)$ and $\left.n=44,108(2018)\right) ;(s)$ Issues considered: (1) guidance on conducting an exam to prevent cervical cancer and (2) guidance on family planning and contraceptive methods; ${ }^{(t)}$ Only users in need of dental prosthesis were considered ( $n=5,888(2012)$ and $n=6,353(2018)$ ). 


\section{DISCUSSION}

For PC to become effectively a space capable for offering comprehensive health care, it is essential that it can act as the preferred point of first regular contact of users with the health system, exercising the function of coordinating care in the Health Care Networks Health, especially for those users who demand the most recurrent services ${ }^{(4-5)}$.

With regard to the provision of programmed actions for population groups, historically identified as priorities, the data reveal that, although there has been an important increase in the proportion of teams that ensure preventive and assistance care for such groups, quality of care still faces great challenges mainly in the north and center-west. Considering the marking elements of quality of care, as shown by data, only $22.4 \%$ of mothers stated that they were asked about how they were feeling emotionally, received contraceptive methods, gynecological care and had their breasts examined. For children up to 2 years old, about $1 / 3(32.7 \%)$ received care during the first week of life, underwent the heel prick test, had their vaccination up to date, among other essential issues in monitoring children in this age group. Among users with diabetes, only about $1 / 3$ reported having taken a blood glucose test, having their feet examined by a health professional and having received guidance on foot care in the past 6 months.

Services organized to meet the most recurring demand in a qualified manner necessarily imply better health outcomes, especially for users in chronic conditions, avoiding aggravation and deterioration of their health and search for specialized services and hospitalizations for preventable causes by $P C^{(9,24)}$. As literature points out, all of this requires eliminating physical and organizational barriers to access, availability of inputs and equipment that allow developing actions and qualifying professionals to develop good care practices ${ }^{(25)}$.

Health promotion actions combined with efforts to prevent diseases have occupied a prominent position in practices undertaken by FHS since its inception. Teams seek in a diversified way to articulate care-curative actions with practices that aim to prevent illness and contribute to establishing adequate living and health conditions in their territories of activity ${ }^{(11-12)}$ - either through activities in programmatic groups and health education, coordinated initiatives with other public or civil society organizations or direct interventions during individual contact with users at the time of service ${ }^{(12)}$.

As the data presented suggest, in 2018, almost all teams developed promotion and prevention actions (96.2\%)/ however, when selecting a specific theme to capture users' perception regarding the scope of cervix cancer prevention actions and family planning, it is noted that there are still important gaps to be filled by the teams. The qualification of promotion and prevention initiatives requires changes in the conception and organization of the work carried out at $\mathrm{BHU}$ and must overcome a prescriptive aspect of behaviors considered to be healthy, in the perspective of building, based on permanent agreement between professionals and users, possibilities for changes in individuals' attitudes and habits ${ }^{(12)}$.

When it comes to collecting material for laboratory exams and procedures and minor surgeries, the data revealed that over the years 2010 there was an important increase in the prevalence of teams that started to regularly offer an essential set of procedures such as drainage of abscess, suture of wounds, removal of stitches, dressings, among others. A recurring criticism of PC is that its ability to solve users' problems is very low, and this negative assessment often results from lack of acceptance of spontaneous demand and the limited offer of procedures that are often demanded by users, greatly reducing the possibility for the team to solve problems in BHU itself without the need for referrals to other specialties in other points of care.

As the literature points out, which has been focusing more recently on the scope of practices of medical professionals and nurses who are part of $\mathrm{FH}$ teams, great obstacles still persist for the expansion of the offer of a strategic set of actions that would allow an increase in PC resolution ${ }^{(13-14)}$. Often, teams do not offer or perform procedures due to lack of training to ensure the necessary skills for their proper technical execution, due to the lack or scarcity of materials and inputs essential for their performance, or even because they do not understand $\mathrm{PH}$ as the place for offering these shares ${ }^{(13-14)}$. The restriction of the scope of procedures offered/performed in $\mathrm{BHU}$ is even greater in more populous cities that have a network of emergency and specialized services in greater number. In 2018, while in municipalities with up to 10,000 inhabitants the proportion of teams that performed all the procedures listed was $64.9 \%$, in the largest cities it was 25.5\% (Table 1).

Regarding the offer of basic oral health procedures, we noticed that, in general, there were no relevant improvements in the prevalence of teams that started to offer procedures such as application of sealant, topical application of fluoride, amalgam restoration, composite resin restoration and tooth extraction. It was mainly in medium-sized cities that this setting evolved most positively, and northern Brazil continued to show results much lower than the other regions, clearly demonstrating the degree of difficulty that municipalities in the region encounter in organizing adequate oral health offers. Looking at the issue from another angle, considering users' response, when selecting an important marker of the quality of the oral health offer, such as availability and monitoring for the proper use of dental prosthesis, the results indicate low public access in BHU and users still need to go to private clinics and offices to access prostheses when needed.

Oral health offer is an important element of the Brazilian experience, related to comprehensiveness in PC. However, as literature points out, the population coverage of oral health remains lower than FHS coverage. Municipalities still find it difficult to equip and supply the units with essential inputs for oral health actions and the organization of the network continues with a high degree of fragmentation and disarticulation, especially when it comes to the follow-up of users diagnosed with oral cancer and who need dental prosthesis ${ }^{(15-16)}$.

Another important issue associated with the ability of PC to enhance its offer of comprehensive care is the organization of the teams' agenda for carrying out actions in people's and families' homes, especially when it comes to the longitudinal monitoring of users with chronic non-communicable diseases and users more susceptible to illness or worsening of diseases due to socioeconomic factors ${ }^{(17-18)}$. Caring for home care can provide greater 
knowledge about users, considering their habits, routine and risk factors under which they are exposed, contributing to a more focused attention on their concrete needs ${ }^{(17-18)}$. For this theme, as data suggest, most teams seek to organize their work process in order to ensure that home visits are carried out and that those visits are determined by users' risk and vulnerability. At the same time, most users interviewed indicated that they receive a visit from $\mathrm{CHW}$ at their home, although the percentages decreased between 2012 and 2018, mainly in the center-south and in most populous cities. A possible explanation for this decrease, to be better investigated, is that, as of 2015, the number of $\mathrm{CHW}$, for the first time since the implementation of the Community Health Workers Program (PACS - Programa de Agentes Comunitários de Saúde), has decreased, leading to falls successive coverage ${ }^{(6)}$.

Seeking integrality must necessarily incorporate a broader spectrum of knowledge that requires overcoming the hegemonic medical model, which favors a biological perspective of health problems and needs, restricting care strategies to their biological aspect, neglecting their interdependence with social, economic and cultural factors ${ }^{(17,20)}$. In this sense, the articulation of different knowledge, in favor of expanding the services' capacity to find appropriate responses to the singularity of individuals, requires approaches that favor the multidimensionality of care and the overcoming of compartmentalized and medicalizing practices ${ }^{(20,26)}$. Initiatives such as NASF implementation and the incorporation of a broader range of medical rationales in care practices that occur at $\mathrm{BHU}$ and in the territory where $\mathrm{FH}$ teams operate can represent an important accumulation for the achievement of assumptions that permeate the idea of comprehensiveness in $\mathrm{PC}^{(19-21)}$.

The results presented show that there has been a significant increase in the proportion of teams that now have the support of NASF, mainly in smaller cities, due to the change in the financing policy of the $\mathrm{MoH}$, which, since the end of 2012, has come to recognize the possibility implementation of NASF in municipalities where there were only 1 or $2 \mathrm{FH}$ teams. However, as literature points out, many challenges still arise for individuals' qualification and workers' collective actions who integrate $\mathrm{NASF}^{(19)}$. They find it very difficult to consolidate their bond with other professionals and the population, mainly due to the high number of $\mathrm{FH}$ teams linked to each nucleus, obstacles to their movement between the territories where the different teams operate and the lack of adequate training and skills to work at $\mathrm{NASF}^{(19)}$. The results also showed a significant increase in the prevalence of teams that offer ICP as a strategy to expand the therapeutic range available to users looking for $\mathrm{BHU}$, and the highest percentages remain in the Southeast and larger cities, locations that concentrate the largest portion of ICP training centers. In most situations, it is the workers themselves who take the initiative to seek training, given that their offer in Brazil is still insufficient, diffuse and with limited quality ${ }^{(21)}$.

Finally, the effectiveness of comprehensiveness in PC also requires the health service to make an effort to better understand the reality of the territory in which it operates and the ability to connect with other sectors of public power and organized civil society. A greater articulation of PC with educational, social assistance, cultural, religious and associative apparatus, in general, shifts the axis of performance of services to a more comprehensive perspective of understanding users' needs, considering that most of the elements that produce an effect on individuals' and communities' health occurs in the territory where they live and form their bonds of coexistence and daily interaction ${ }^{(12,22)}$. A good example of intersectoral action between $\mathrm{FH}$ and education is the Health at School Program (PSE - Programa Saúde na Escola), which, according to the results, also showed an increase in the analyzed period, evidencing the growing effort of managers and workers to implement intersectorial actions, regardless of the observed context.

\section{Study limitations}

Although most of the existing $\mathrm{FH}$ teams in the country participated in the PMAQ, as it is a membership program, the sample of teams that make up the study is not completely random. At the same time, as there were changes in instruments between the $1^{\text {st }}$ and $3^{\text {rd }}$ cycles, important issues related to comprehensiveness, which could increase the depth of the analyzes undertaken, could not be mobilized, such as issues associated with practices carried out in households and reasons that embarrass or limit the possibility for teams to offer a broader scope of actions.

\section{Contributions to nursing, health, and public policy}

The results of this study can contribute to improving and reorientating policies, programs and practices of health workers, including nursing professionals, directing them to face aspects of comprehensiveness that present greater gaps and deficiencies, with a look at the different contexts for developing actions that can meet the specificities of the different realities.

\section{CONCLUSIONS}

Access and comprehensiveness of care in PC has been strengthening since the Brazilian State, through SUS, passed and implemented more structured PC policies across the country. As we can see throughout the study, for almost all dimensions related to comprehensiveness that were addressed, improvements were found during the analyzed period. There was an increase in the prevalence of $\mathrm{FH}$ teams that provide preventive and assistance care for priority population groups; almost all of them develop promotion and prevention actions; there was an increase in the prevalence of teams that began to regularly offer an essential set of procedures; most of them organize their work process to ensure that home visits are carried out, with priority for users at greatest risk and vulnerability; expressive growth was found in the proportion of teams that started to count on NASF support and that incorporated ICP in the list of offers to users, in addition to the increase in the percentage of teams that seek to carry out intersectoral actions through articulation with the education sector.

On the other hand, the data also revealed important challenges for qualification of PC service offer. For groups such as puerperal women, children up to 2 years old and users with diabetes, there was a need to improve team practices to ensure adequate care for users, especially in northern and center-western Brazil. Likewise, important gaps were identified for the qualification of preventive 
actions aimed at women's health and obstacles to expanding the offer of a strategic set of procedures that would allow greater capacity for resolving PC, especially in the southeast and more populous municipalities. As for oral health offer, significant bottlenecks also persist, mainly in the north, which continue to present results much lower than the other regions. The percentage of users who said they received a visit from $\mathrm{CHW}$ showed a small negative fluctuation, with greater falls in the center-south and more populous cities.

This improvement setting depended on the direct action of the State from the implementation of actions aimed at overcoming historical obstacles experienced by SUS and PC, such as investment in the structuring of $\mathrm{BHU}$ and in processes to increase access and improve quality, as well as provision of medical professionals in areas of low medical workforce attraction and fixation. However, the fiscal austerity agenda that has been implemented over the past few years has been imposing on social policies, including health, a movement of retraction of available resources, representing a strong harbinger of a decrease in the State's capacity to respond to the needs of population's health and, consequently, the possibility that PC will follow a positive path of progress towards a greater offer of comprehensive care.

\section{REFERENCES}

1. Pinheiro R, Ferla A, Silva Jr AG. Integrality in the population's health care programs. Ciênc Saúde Colet. 2007;12(2):343-49. https://doi. org/10.1590/S1413-81232007000200010

2. Noronha JC, Lima LD, Machado CV. O Sistema Único de Saúde - SUS. In: Giovanella L, Escorel S, Lobato LVC, Noronha JC, Carvalho Al, (Orgs.). Políticas e sistema de saúde do Brasil. Rio de Janeiro: Editora Fiocruz; 2012. p. 365-93.

3. Mattos RA. [Comprehensiveness in practice (or, on the practice of comprehensiveness)]. Cad Saúde Pública. 2004;20(5):1411-16. https://doi. org/10.1590/S0102-311X2004000500037 Portuguese.

4. Carnut L. [Care, integrality and primary care: essential articulation to reflect on the health sector in Brazil]. Saúde Debate. 2017;41(115):117786. https://doi.org/10.1590/0103-1104201711515 Portuguese.

5. Giovanella L. Atenção primária à saúde seletiva ou abrangente? Cad Saúde Pública. 2008;24(Suppl-1):S21-S23. https://doi.org/10.1590/ S0102-311X2008001300005

6. Ministério da Saúde (BR). Histórico de cobertura da atenção básica [Internet]. 2019[cited 2019 Nov 14]. Available from: https://egestorab. saude.gov.br/paginas/acessoPublico/relatorios/relHistoricoCobertura.xhtml

7. Dourado I, Medina MG, Aquino R. The effect of the Family health strategy on usual source of care in Brazil: data from the 2013 National Health Survey (PNS 2013). Int J Equity Health [Internet]. 2016 [cited 2020 Aug 07];15:151. Available from: https://equityhealthj. biomedcentral.com/track/pdf/10.1186/s12939-016-0440-7

8. Macinko J, Mendonça CS. [The family health strategy, a strong model of primary health care that delivers results]. Saúde Debate [Internet]. 2018 [cited 2020 Aug 07];42(spe-1):18-37. https://doi.org/10.1590/0103-11042018s102 Portuguese.

9. Bastos LS, Assis MMA, Nascimento MAA, Oliveira LCF. [Integrality-building in the care of people with diabetes mellitus in a healthcare unit of Feira de Santana, Bahia State]. Ciênc Saúde Colet. 2011;16(Suppl-1):1417-26. https://doi.org/10.1590/S1413-81232011000700077 Portuguese.

10. Sala A, Luppi CG, Simões O, Marsiglia RG. [Integrality and primary health care: assessment in the perspective of health services users in the city of São Paulo]. Saúde Soc. 2011;20(4):948-60. https://doi.org/10.1590/S0104-12902011000400012 Portuguese.

11. Arce VAR, Sousa MF. [Comprehensive care: social representations of Family health teams in Distrito Federal, Brazil]. Saúde Soc. 2013;22(1):109-23. https://doi.org/10.1590/S0104-12902013000100011 Portuguese.

12. Prado NMBL, Santos AM. [Health promotion in primary health care: systematization of challenges and intersectoral strategies]. Saúde Debate. 2018;42(spe1):379-95. https://doi.org/10.1590/0103-11042018s126 Portuguese.

13. Girardi SN, Carvalho CL, Pierantoni CR, Costa JO, Stralen ACS, Lauar TV, et al. Assessment of the scope of practice of physicians participating in the Mais Médicos (More Doctors) Program, and associated factors. Ciênc Saúde Colet [Internet]. 2016 [cited 2020 Aug 07];21(9):2739-48. https://doi.org/10.1590/1413-81232015219.15912016

14. Girardi SN, Stralen ACS, Lauar TV, Cella JN, Araújo JF, Pierantoni CR, et al. Scope of practice in primary care: physicians and nurses in five health regions in Brazil. Rev Bras Saúde Matern Infant. 2017;17(suppl-1):S171-S184. https://doi.org/10.1590/1806-9304201700s100008

15. Sanchez HF, Werneck MAF, Amaral JHL, Ferreira EF. [Integrality in everyday dental care: review of the literature]. Trab Educ Saúde. 2015;13(1):201-14. https://doi.org/10.1590/1981-7746-sip00020 Portuguese.

16. Casotti E, Contarato PC, Fonseca ABM, Borges PKO, Baldani MH. [Dental care in Brazil: an analysis based on PMAQ-AB external evaluation]. Saúde Debate. 2014;38(esp):140-57. https://doi.org/10.5935/0103-1104.2014S011 Portuguese.

17. Comes Y, Trindade JS, Pessoa VM, Barreto ICHC, Shimizu HE, Dewes D, et al. The implementation of the Mais Médicos (More Doctors) Program and comprehensiveness of care in the Family Health Strategy. Ciênc Saúde Colet. 2016;21(9):2729-38. https://doi. org/10.1590/1413-81232015219.15472016

18. Gasparini MFV, Furtado, JP. Longitudinality and comprehensiveness on the more doctors program: an evaluative study. Saúde Debate. 2019;43(120):30-42. https://doi.org/10.1590/0103-1104201912002 
19. Araújo Neto JD, Albuquerque IMN, Lira GV, Bosi MLM. [Restrictive aspects of comprehensive healthcare in the family health supporting nuclei: the stakeholder's viewpoint]. Physis. 2018;28(4):1-25. https://doi.org/10.1590/s0103-73312018280417 Portuguese.

20. Pinheiro R, Luz MT. Práticas eficazes x modelos ideias: ação e pensamento na construção da integralidade. In: Pinheiro R, Mattos RA, (Orgs.). Construção da integralidade: cotidiano, saberes e práticas em saúde. 5. Ed. Rio de Janeiro: CEPESC/UERJ/Abrasco; 2010. p. 9-36.

21. Tesser CD, Sousa IMC, Nascimento MC. [Traditional and complementary medicine in primary health care in Brazil]. Saúde Debate. 2018;42(spe-1):174-88. https://doi.org/10.1590/0103-11042018s112 Portuguese.

22. Santos CTB, Barros IS, Amorim ACCLA, Rocha DG, Mendonça AVM, Sousa MF. Integrality in Brazil and Venezuela: similarities and complementarities. Ciênc Saúde Colet. 2018;23(4):1233-40. https://doi.org/10.1590/1413-81232018234.16122016

23. Ministério da Saúde (BR). Microdados da avaliação externa do Programa Nacional de Melhoria do Acesso e da Qualidade da Atenção Básica (PMAQ) [Internet]. 2019 [cited 2019 Sep 28]. Available from: https://aps.saude.gov.br/ape/pmaq

24. Ceccon RF, Meneghell SN, Viecili PRN. Hospitalization due to conditions sensitive to primary care and expansion of the Family health program in Brazil: an ecological study. Rev Bras Epidemiol. 2014;17(4):968-77. https://doi.org/10.1590/1809-4503201400040014

25. Tomasi E, Cesar MADC, Neves RG, Schmidt PRC, Thumé E, Silveira DS, et al. Diabetes care in Brazil; program to improve primary care access and quality (PMAQ). J Ambul Care Manage[Internet]. 2017 [cited 2020 Aug 07];40(sup2):S12-S23. Available from: https://journals.Iww.com/ ambulatorycaremanagement/Fulltext/2017/04001/Diabetes_Care_in_Brazil_Program_to_Improve.3.aspx

26. Campos GWS. [Local reference teams and specialized matrix support: an essay about reorganizing work in health services]. Ciênc Saúde Colet. 1999;4(2):393-403. Available from: doi: https://doi.org/10.1590/S1413-81231999000200013 Portuguese. 